Belmonte Almagro, M.L., García Sanz, M.P. \& Galián Nicolás, B. (2016). Blogfolio. Aprendiendo a través de la evaluación. Revista Electrónica Interuniversitaria de Formación del Profesorado, 19(3), 25-33.

\title{
Blogfolio. Aprendiendo a través de la evaluación
}

\author{
Ma Luisa Belmonte Almagro, Ma Paz García Sanz, Begoña Galián Nicolás \\ (Universidad de Murcia, Murcia, España)
}

\section{Resumen}

Una de las herramientas más innovadoras utilizadas en la web 2.0 es el denominado blogfolio. Con el fin de determinar las ventajas y limitaciones de dicha herramienta en el ámbito educativo, en esta investigación, de carácter evaluativo, se ha querido conocer la opinión de un grupo de estudiantes del Grado en Educación Infantil de la Universidad de Murcia, sobre un blogfolio que los mismos estaban utilizando durante el transcurso de una de las asignaturas. Para ello, se ha empleado un cuestionario ad hoc, validado mediante el procedimiento interjueces. Los resultados indican que, en general, el blogfolio es adecuado como instrumento para el aprendizaje y la evaluación de los estudiantes, así como para fomentar el trabajo colaborativo entre los mismos. Si bien se han hallado diferencias significativas en lo que respecta a la percepción que tienen los alumnos y alumnas sobre la utilización del portafolios en función del grupo al que éstos pertenecen, no ha sido así considerando el conocimiento previo que los estudiantes tenían sobre los blogs, ni en función de que hubiesen creado antes otros blogs, ni tampoco según hiciesen seguimiento o no de algún blog.

\section{Palabras clave}

Evaluación; aprendizaje; tecnologías de la información y la comunicación; grado universitario. 


\title{
Blogfolio. Learning through evaluation
}

\begin{abstract}
One of the most innovating tools used in web 2.0 is the blogfolio. This research, of evaluational nature, has the aim of determining the advantages and limitations of said tool in the educational field. For that purpose, a group of Pre-school Education degree students from the University of Murcia were asked about their opinion on a blogfolio that they had been using throughout the course of one of their subjects. An ad hoc questionnaire, validated by inter-judge reliability, was used for that matter. The results show that, in general, the blogfolio is suitable as an instrument for the students' learning process and as a form of evaluation, as well as a way of encouraging collaborative work among them. While meaningful differences have been found regarding the students' perception of the portfolio depending on the group they belonged to, no significant differences based on previous knowledge about blogs (either by having followed one or having created one) have been noticed.
\end{abstract}

\section{Key words}

Evaluation; learning; information and communication technologies; academic degree.

\section{Introducción}

De forma tradicional, la enseñanza universitaria se ha basado en un modelo metodológico centrado en el docente, donde la transmisión de contenidos y su posterior reproducción por el alumnado, la lección magistral y el trabajo individual, han sido su principal eje.

En la actualidad, enseñar a través de las Tecnologías de la Información y la Comunicación (TIC), exige, tanto por parte del profesorado como por la del discente, una serie de cambios que suponen un alejamiento de este modelo, favoreciendo un avance hacia la calidad de la educación (Aguaded, López Meneses y Alonso Díaz, 2010). Estos cambios que se vienen sucediendo han generado una sociedad más informatizada, ya que nunca se había visto tan influenciada por las nuevas tecnologías como en los últimos años (Cabero, 2001).

En la actual transformación didáctica originada por la incorporación de las universidades españolas al Espacio Europeo de Educación Superior (EEES), uno de los objetivos es el de establecer un sistema de enseñanza que favorezca la formación integral del estudiante, con el fin de dar respuesta a las necesidades de la tan exigente y efímera realidad sociolaboral que demanda nuevos perfiles competenciales y conocimientos permanentemente actualizados a lo largo del ciclo vital.

La aparición de entornos interactivos 2.0, y las herramientas como los blogs, wikis, la realidad aumentada, redes sociales y demás medios de información y comunicación emergentes, propician un contexto idóneo para el desarrollo de competencias como el pensamiento crítico, la autonomía, la iniciativa, el trabajo colaborativo y la responsabilidad individual, todas ellas claves en el actual EEES (Cabero, López Meneses, y Llorente, 2012). Estas nuevos instrumentos se convierten en útiles métodos para la recogida de información, en tanto que permiten reflejar los puntos fuertes y débiles del estudiante, documentando su avance de manera paulatina y permitiendo diseñar la enseñanza futura en función del feedback recibido (Bulwik, 2004). 
Una de las herramientas más innovadoras utilizadas en la web 2.0 es el denominado Blogfolio, considerado como una adaptación del concepto e-portafolio utilizado en formato blog. Con contenido específicamente educativo, se trata de una herramienta dinámica para la evaluación y el aprendizaje en la que el alumnado muestra una selección de su producción académica, a la vez que reflexiona sobre ella a través de una conversación con el resto de estudiantes y con el docente (García Carreño, 2010, 2011). Por lo tanto, el blogfolio es el resultado de la unión de dos sistemas de reflexión y recogida de información, como son el blog y el portafolio, que aun a pesar de sus diferencias, ambas potencian la autonomía y la libertad del alumnado en su proceso de aprendizaje y (auto)evaluación, siendo éstas dos características básicas e indispensables de esta nueva herramienta reflexiva.

Así pues, el blogfolio es considerado un método de transmisión y valoración de conocimientos que centra su atención en el estudiante, ofreciendo la posibilidad de estimar sus capacidades, habilidades y estrategias en el marco de una disciplina concreta. Todo ello facilita la interacción social entre docente y discentes, vehiculando la comunicación de forma fluida, tanto para el desarrollo de las tareas de tutorización y seguimiento, como para el trabajo colaborativo entre estudiantes (Area y Adell, 2009). Así, constituye una herramienta pedagógica útil tanto para el desarrollo de competencias de aprendizaje como para la evaluación de los diferentes niveles de logro con los que éstas se pueden adquirir (Colás, Jimenez y Villaciervos, 2005).

La evaluación constituye parte de los procesos educativos, favoreciendo la reflexión y la autorregulación desde el principio hasta el final (Álvarez, 2008). Más concretamente, la evaluación formativa está cobrando en la actualidad un protagonismo tal que ya no se habla únicamente de evaluar el aprendizaje, sino, cada vez con más fuerza, de una evaluación para el aprendizaje, la cual, según Gessa (2011), se apoya en tres aspectos fundamentales: plantear las tareas de evaluación como tareas de aprendizaje, involucrar a los estudiantes en la evaluación y ofrecer los resultados de la evaluación a modo de feedbak.

Así, esta herramienta evaluativa resulta especialmente conveniente dado que se realiza a partir de la participación activa de todos los implicados, especialmente del alumnado universitario (Bretones, 2008; Taras, 2010; Trevitt, Breman y Stocks, 2012). La implicación de los estudiantes en su propia evaluación les permite llevar a cabo procesos de autoreflexión, que a su vez, según Nicol y Macfarlane-Dick (2006), conllevan mecanismos de retroalimentación en su aprendizaje. Además, cuando el blogfolio se utiliza para la evaluación formativa, tiene el potencial de mejorar la autoestima del estudiante (Barrett, 2006).

Este instrumento de evaluación es especialmente pertinente por su gran capacidad para evaluar las competencias desarrolladas por el estudiante y por el protagonismo que otorga al alumnado en su proceso de aprendizaje y evaluación (Rodríguez Sánchez, 2011). Constituye un documento interactivo y novedoso que refleja un trabajo continuado de reflexión y orientación, que además puede ser consultado y evaluado por el docente, a tiempo real.

Desde el punto de vista didáctico, la incorporación del blogfolio al día a día del alumnado puede servir como instrumento que ayude al estudiante a estar más orientado, a organizar sus ideas y propósitos académicos, facilitándole su propia evaluación de manera diaria a partir de todas aquellas evidencias que selecciona. Para Roig (2009), se trata de una herramienta sencilla de utilizar, muy intuitiva, en la que los contenidos en una plataforma blog cuentan con una cronología muy estructurada, permitiendo ser analizados inmediatamente después de ser introducida cualquier modificación. No obstante, a pesar 
de las ventajas que conlleva su utilización, no está exento de dificultades, fundamentalmente porque supone un esfuerzo añadido, tanto para el alumnado como para el profesorado (Galán Mañas, 2015).

En este escenario, lo que se pretende con esta investigación es analizar la percepción de los estudiantes universitarios acerca de la utilización del blogfolio educativo como herramienta para evaluar su trabajo, teniendo en cuenta sus respuestas en función del grupo al que pertenecen y de su experiencia previa con dicha herramienta. Todo ello en mitad del proceso formativo, para experimentar los cambios durante el desarrollo de la asignatura, considerando los resultados que se vayan obteniendo.

\section{Método}

Nos encontramos ante una investigación evaluativa, concretamente en la fase referida a la evaluación formativa dentro del denominado "Ciclo de intervención educativa" (García Sanz, 2012, p.167).

De una población de 192 estudiantes pertenecientes a los tres grupos de segundo curso del Grado en Educación Infantil de la Universidad de Murcia, que cursaban la asignatura de Medios, Materiales y TIC para la Enseñanza, en el estudio han participado 133 universitarios (tamaño muestral necesario: 129). La estrategia de muestreo ha sido no probabilística de voluntarios, ya que los alumnos han decidido colaborar voluntariamente.

Para la recogida de información se ha utilizado un cuestionario ad hoc de 24 ítems, elaborado y validado para la investigación. Concretamente, la validez de contenido se obtuvo mediante el procedimiento interjueces y para la fiabilidad, se calculó el coeficiente Alfa de Cronbach, apreciándose una elevada consistencia interna del instrumento $(\alpha=, 965)$.

Los 4 primeros ítems del cuestionario constituyen las variables predictoras del estudio (grupo de pertenencia del alumnado, conocimiento, creación y seguimiento de blogs. Estas tres últimas variables se han agrupado en otra nueva denominada: experiencia previa de los estudiantes con los blogs). Los 20 ítems siguientes, específicos del tema a investigar, conforman las variables criterio, los cuales integran una escala numérica tipo Likert de 5 grados. A continuación, y para finalizar el instrumento, se incluye un apartado abierto de observaciones para matizar cualitativamente, en caso de que fuera necesario, la puntuación numérica otorgada.

Respecto al procedimiento de la investigación, de acuerdo con los docentes responsables de la asignatura, se les pidió a los estudiantes que de manera online, respondieran el cuestionario, de forma voluntaria y anónima.

Una vez recogida la información, ésta fue analizada utilizando el paquete estadístico SPSS, versión 19, tanto para los análisis descriptivos como inferenciales $(\alpha=, 05)$. Se hizo uso de la estadística no paramétrica, por no cumplirse los requisitos para poder aplicar la estadística paramétrica.

\section{Resultados}

En relación a la valoración del blogfolio por los estudiantes, en la Tabla 1 se muestran las medias y desviaciones típicas de las variables criterio de la investigación.

Tabla 1.

Estadísticos descriptivos de la valoración del blogfolio. 


\begin{tabular}{|l|c|c|}
\hline \multicolumn{1}{|c|}{ Ítems } & Media & $\begin{array}{c}\text { Desviación } \\
\text { típica }\end{array}$ \\
\hline Global & 3,78 &, 742 \\
\hline Estructura y organiza las tareas & 3,89 &, 881 \\
\hline Refuerza la comprensión de contenidos & 3,74 &, 982 \\
\hline Desarrolla conceptos, habilidades y actitudes & 3,70 &, 904 \\
\hline Focaliza la atención en lo más relevante & 3,83 &, 900 \\
\hline Informa sobre el aprendizaje adquirido & 3,62 &, 967 \\
\hline Asegura una evaluación transparente & 3,77 &, 976 \\
\hline Permite una evaluación continua y formativa & 3,83 &, 963 \\
\hline Conlleva la colaboración docente-estudiante & 3,98 &, 957 \\
\hline Permite enriquecerse de otros compañeros/as & 3,97 &, 953 \\
\hline Favorece la autonomía del estudiante & 3,89 &, 940 \\
\hline Desarrolla el pensamiento reflexivo y crítico & 3,68 &, 932 \\
\hline Asegura aprendizajes mínimos y su ampliación & 3,66 &, 912 \\
\hline Motiva al estudiante a aprender & 3,69 & 1,088 \\
\hline Refuerza la formación en TIC & 4,06 &, 903 \\
\hline Fomenta la imaginación y la creatividad & 4,09 &, 857 \\
\hline Permite la evaluación objetiva y fiable & 3,55 &, 957 \\
\hline Es eficiente la relación tiempo/utilidad & 3,47 & 1,027 \\
\hline Resulta fácil de usar & 3,56 & 1,083 \\
\hline Ayuda al desarrollo de competencias específicas & 3,74 &, 893 \\
\hline Es útil para el maestro/a de Educación Infantil & 3,91 & 1,018 \\
\hline
\end{tabular}

Como se aprecia en la Tabla 1, la valoración global del blogfolio por parte del alumnado ha sido muy cercana a alta, superando todos los ítems el estándar considerado (3). Destacan como aspectos mejor valorados los referidos a que el blogfolio fomenta la imaginación y la creatividad (Media=4,09), refuerza la formación en TIC (Media=4,06), permite enriquecerse de otros compañeros/as (Media=3,97) y es útil para el maestro/a de Educación Infantil (Media=3,91). Por el contrario, aun dentro de una puntuación medio-alta, los ítems peor valorados por los estudiantes han sido los que hacen referencia a que en el blogfolio es eficiente la relación tiempo/utilidad (Media=3,47), la herramienta permite la evaluación objetiva y fiable (Media=3,55) y resulta fácil de usar (Media=3,56). Precisamente, como muestra la Tabla 1, estos tres ítems arrojan desviaciones típicas altas, lo que pone de manifiesto la variedad de opiniones de los estudiantes respecto a la media (coeficientes de variación de $28,93 \%, 26,96 \%$, y $30,42 \%$, respectivamente).

Respecto a la valoración del blogfolio en función del grupo de pertenencia de los estudiantes (cada uno impartido por un docente diferente), la Tabla 2 muestra las medias 
obtenidas en cada uno de los grupos, así como el valor de Chi-cuadrado y su correspondiente significación estadística.

Tabla 2.

Medias de cada grupo y resultados de la prueba de Kruskal-Wallis en función del Grupo-clase.

\begin{tabular}{|c|c|c|c|}
\hline Grupo & Media & Chi-cuadrado & Significación \\
\hline 1 & 3,42 & & \multirow{2}{*}{14,49} \\
\cline { 1 - 2 } 2 & 4,04 & & \multirow{2}{*}{, 001} \\
\hline 3 & 3,73 & & \\
\hline
\end{tabular}

En la Tabla 2 se observa la existencia de diferencias significativas entre los tres grupos de estudiantes en lo que respecta a la valoración que éstos han realizado del blogfolio. Concretamente, dichas diferencias se encuentran entre los grupos 1 y $2(P=, 000)$, a favor de este último, y 2 y $3(P=, 020)$, a favor del primero, pero no entre los grupos 1 y $3(P=, 125)$.

En cuanto a las puntuaciones otorgadas por los estudiantes, considerando su experiencia previa con los blogs, la Tabla 3 señala las medias obtenidas en cada uno de los grupos configurados a partir del conocimiento, creación y seguimiento previo que el alumnado posee acerca de dicha herramienta. En esta tabla, también se concreta el valor de la $U$ de Mann-Whitney y su correspondiente significación estadística.

Tabla 3.

Medias de cada grupo y resultados de la prueba de $U$ de Mann-Whitney, en función de la experiencia previa del alumnado con Blogs.

\begin{tabular}{|c|c|c|c|}
\hline \multicolumn{4}{|c|}{ Conocimiento previo de lo que es un blog } \\
\hline Grupo & Media & U de Mann-Whitney & Significación \\
\hline NO & 3,97 & \multirow{2}{*}{582} & \multirow{2}{*}{,778 } \\
\hline Sí & 3,77 & & \\
\hline \multicolumn{4}{|c|}{ Creación previa de blogs } \\
\hline NO & 3,91 & \multirow{2}{*}{1883} & \multirow{2}{*}{ 174 } \\
\hline Sí & 3,62 & & \\
\hline \multicolumn{4}{|c|}{ Seguimiento de blogs } \\
\hline NO & 3,79 & \multirow{2}{*}{1583} & \multirow{2}{*}{,862 } \\
\hline Sí & 3,74 & & \\
\hline
\end{tabular}

La Tabla 3 indica que, a nivel muestral, los estudiantes que no tienen conocimiento previo de lo que es un blog, no lo han creado nunca y no realizan el seguimiento de ninguno han valorado mejor el blogfolio que los que sí tienen una experiencia previa con blogs, en relación a las tres variables consideradas. Sin embargo, la prueba $U$ de Mann-Whitney muestra que dicha valoración que los estudiantes han otorgado al blogfolio no se encuentra 
significativamente en función de la experiencia previa que los mismos tienen respecto a los blogs $-n i$ en conocimiento $(P=, 778)$, ni en creación anterior $(P=, 174)$, ni en seguimiento $(P=, 862)$.

\section{Discusión y conclusiones}

En los últimos años, el uso de las TIC en la universidad es un condicionante prioritario en la formación de los estudiantes (Oblinger y Oblinger, 2005), donde la aparición de la web 2.0 ha propiciado una mejora de la interacción entre los usuarios. Esto ha generado grandes ventajas (Fainholc, 2008), así como un contexto idóneo para el protagonismo del estudiante y el desarrollo de competencias muy valoradas en la actualidad (Cabero, López y Llorente, 2012), en el que el docente es ante todo, un creador de oportunidades de aprendizaje (Morales, 2006).

En esta investigación, en general, se ha puesto de manifiesto la adecuación del blogfolio como instrumento de evaluación del aprendizaje de los estudiantes. Estos resultados coinciden con los encontrados por Aguaded, López Meneses y Alonso Díaz (2010), quienes concretaron que los blogs educativos son fáciles de usar, animan a los estudiantes a asumir un papel activo y constituyen instrumentos útiles para la autoevaluación. Asimismo, Salinas y Viticcioli (2008) concluyeron que la evaluación realizada por los estudiantes sobre el blog utilizado confirmó su idoneidad como recurso para la innovación, resaltando la mejora que el mismo proporciona para la comprensión de la materia y el deseo de que su uso fuera extendido a otras asignaturas. Igualmente, Roig (2009), refiriéndose al blogfolio, diferencia entre el "continente" (blog) y el "contenido" (porfolios), aportando como principales ventajas del blog su estructura cronológica, su facilidad de uso, su amplia difusión, su interactividad y la consulta permanente del mismo. Las ventajas que esta autora adjudica al portafolios las resume en la flexibilidad de su estructura, el aprendizaje que proporciona y la visión global del proceso educativo desarrollado.

El hecho de que existan diferencias significativas, respecto a la valoración otorgada al blogfolio por los estudiantes entre algunos de los grupos-clase (1 y 2; 2 y 3) puede ser debido a variables que afectan tanto al docente (explicación de la utilización de la herramienta, insistencia en su empleo, seguimiento y valoración de la misma, etc.), como al propio alumnado (grado de motivación en el empleo de TIC, expectativas académicas, enfoque de aprendizaje, etc.). Por ello, se debe continuar investigando en su utilización en el contexto universitario para ir mejorando progresivamente su uso.

Por otra parte, a pesar de no existir diferencias estadísticamente significativas en relación a las valoraciones otorgadas por los estudiantes al blogfolio, en función de ninguna de las tres variables que conforman la experiencia previa del alumnado con blogs, las puntuaciones medias muestrales han puesto de manifiesto que los discentes que no poseen conocimiento previo de esta herramienta, que no lo han creado nunca y que tampoco realizan ningún seguimiento de blogs, han valorado mejor el blogfolio que los estudiantes que han concedido una respuesta afirmativa a estas tres variables. Esto puede ser debido a que al alumnado sin experiencia previa en blogs, puede haberle impactado más la utilización del blogfolio que a los que ya estaban familiarizados con herramientas similares (blogs).

Para concluir, añadimos que el blogfolio es uno de los instrumentos de evaluación capaz de evaluar las competencias alcanzadas por el alumnado, facilitando las tareas de seguimiento que conducen a la mejora de los procesos educativos, si bien, como se ha comentado, habría que continuar indagando en las variables que pueden influenciar en su utilización, mejorar las instrucciones para su uso, así como su validez y fiabilidad. 


\section{Referencias}

Aguaded, J.I., López Meneses, E. y Alonso Díaz, L. (2010). Innovating with Blogs in University Courses: a Qualitative Study. The New Educational Review, 22 (3-4), 103-115.

Area, M. y Adell, J. (2009). ELearning: Enseñar y aprender en espacios virtuales. En J. De Pablos (Coord). Tecnología Educativa. La formación del profesorado en la era de Internet (pp. 391-424). Málaga: Aljibe.

Álvarez, I. (2008). Evaluación del aprendizaje en la universidad: una mirada retrospectiva y prospectiva desde la divulgación científica. Revista Electrónica de Investigación Psicoeducativa, 6(1), 235-271. Recuperado de http://www.investigacionpsicopedagogica.com/revista/articulos/14/espannol/Art_14_228.pdf

Barrett, H.C. (2006). Using electronic portfolios for formative/classroom-based assessment. Classroom Connect Connected Newsletter, 13(2), 4-6.

Bretones, A. (2008). Participación del alumnado de Educación Superior en su evaluación. Revista de Educación, 347, 181-202.

Bulwik, M. (2004). La evaluación de los aprendizajes y el portafolios. Educación Química, 15(2), 104-107.

Cabero, J. (2001). Tecnología Educativa. Diseño y utilización de medios en la enseñanza. Barcelona: Ediciones Paidós Ibérica, C.A.

Cabero, J., López, E., y Llorente, M. C. (2012). E-Portafolio universitario como instrumento didáctico 2.0 para la reflexión, evaluación e investigación de la práctica educativa en el espacio europeo de educación superior. Virtualidad, Educación y Ciencia, 3(4), 2746.

Colás, P., Jiménez, R. y Villaciervos, P. (2005). Portafolios y desarrollo de competencias profesionales en el marco del Espacio Europeo de Educación Superior. Revista de Ciencias de la Educación, 204, 519-538.

Fainholc, B. (2008). De cómo las TICS podrían colaborar en la innovación socio-tecnológicoeducativa en la formación superior y universitaria presencial. Revista lberoamericana de Formación a Distancia (RIED), 11(1), 53-79.

García Carreño I. (2010). Técnicas para Promover el Aprendizaje Virtual Web 2.0: Aplicaciones del blogfolio. Revista Relada, 4(1), 21-30.

García Carreño, I. (2011). E-Portafolio, rúbricas y blogfolio para una evaluación integral. Revista de Artes y Humanidades UNICA, 12(3), 34-56.

García Sanz, M.P. (2012). Fundamentos teóricos y metodológicos de la evaluación de programas. Murcia: DM.

Morales, P. (2006). Implicaciones para el profesor de una enseñanza centrada en el alumno. Miscelánea Comillas. Revista de Ciencias Humanas y Sociales, 64(124), 11-38. 
Oblinger, D. y Oblinger, J. (Ed.). (2005). Educating the Net Generation. Boulder, CO: Educause. Recuperado de https://net.educause.edu/ir/library/pdf/pub7101.pdf

Galán Mañas, A. (2015). Articulación de la carpeta de aprendizaje en la formación por competencias de traductores. Revista Complutense de Educación, 26(2), 385-403.

Gessa, A. (2011). La coevaluación como metodología complementaria de la evaluación del aprendizaje. Análisis y reflexión en las aulas universitarias. Revista de Educación, 354, 749-764.

Nicol, D., y Macfarlane-Dick, D. (2006). Formative assessment and self- regulated learning: a model and seven principles of good practice. Studies in Higher Education, 31(2), 199218.

Rodríguez Sánchez, M. (2011). Metodologías docentes en el EEES: de la clase magistral al portafolio. Tendencias pedagógicas, 17, 83-103.

Roig, R. (2009). El portafolios a través del blog: conjugando las TIC y la Didáctica. En M.J. Martínez (coord.). El portafolios para el aprendizaje y la evaluación (pp. 171-187). Murcia: Edit.um.

Salinas, I.M. y Viticcioli, S.M. (2008). Innovar con blogs en la enseñanza universitaria presencial. EDUTEC. Revista Electrónica de Tecnología Educativa, 27. Recuperado de http://edutec.rediris.es/Revelec2/revelec27/articulos_n27_PDF/EdutecE_MISanilas_Viticcioli_n27.pdf

Taras, M. (2010). Student self-assessment: processes and consequences. Teaching in Higher Education, 15(2), 199-209.

Trevitt, C., Breman, E. y Stocks, C. (2012). Evaluación y aprendizaje: ¿es ya el momento de replantearse las actividades del alumnado y los roles académicos? Revista de investigación educativa, 30(2), 253-270. 